\title{
THE EFFECT OF USING RETENTION SILICON ON ABUTMENT'S BONE LEVEL AND MOBILITY IN MANDIBULAR OVERDENTURES (RANDOMIZED CLINICAL TRIAL)
}

\author{
Amr Ahmed Naguib*, Ahmed Fayyad ${ }^{* *}$ and Ashraf Abdel-Menaem ${ }^{* * *}$
}

\begin{abstract}
This study was conducted to clinically and radiographically evaluate the effect of retaining mandibular overdenture, by retention silicon material, on the bone level of the abutment teeth. The patients were randomly divided into two groups A and B. All patients were treated by upper conventional complete dentures opposed by tooth supported lower complete overdentures. In group $\mathrm{A}$, the overdenture abutment teeth were covered by post and copping carrying an attachment. Retention Silicon act as housing by relining the denture base in the area of the attachment. In group B, the overdenture abutment teeth were covered by post and copping only with no attachment mechanism. Radiographic evaluation was carried out by measuring the amount of change in alveolar bone height around the abutment teeth. The results of the study showed a significant alveolar bone loss around the abutment teeth in both groups. The group with attachment showed more alveolar bone loss. The amount of bone resorption was nearly 3 times more in the attachment group than the group without attachment by the end of the 6 months follow up period.
\end{abstract}

\section{INTRODUCTION}

An overdenture is a removable dental prosthesis that covers and rests on one or more remaining natural teeth, the roots of natural teeth, and/or dental implants. Also, defined by the glossary of prosthodontics terms as dental prosthesis that covers and is partially supported by natural teeth, natural teeth roots, and/or dental implants. The overdenture is called overlay denture, overlay prosthesis, and superimposed prosthesis. ${ }^{(1)}$
The overdenture prosthesis derives supplementary support and retention from teeth or roots retained under the denture base. Using teeth or roots as abutments enhances overall denture performance ${ }^{(2)}$. Preservation of the reaming natural teeth, when possible, is one of the principles in prosthetic dentistry as long as they do no harm to other oral structures. There are two physiologic reasons related to this therapy the first is concerned with the continued preservation of alveolar bone around the

* Removable Prosthodontics Department Faculty of Oral and Dental Medicine

** Lecturer of Prosthodontics Faculty of Oral and Dental Medicine Cairo University

*** Professor of Prosthodontics Faculty of Oral and Dental Medicine Cairo University 
retained teeth while the second relates to the continued presence of periodontal sensory mechanisms that guide and monitor gnathodynamic functions ${ }^{(3)}$.

Despite the great development of dental implants in the last decades, the principle of preservation of the natural teeth is still valid due to the increased cost of choosing implants as a part of the treatment plan, medical condition of some patients and presence of sufficient amount of bone. On the other hand, the inferior treatment option of complete denture provide less comfort and function and doesn't meet the increased needs of today's patients. The overdenture rose as an acceptable treatment option which can provide support only if the abutment teeth are used without attachment or can provide support and retention for the prosthesis if the abutment teeth carry an attachment mechanism. ${ }^{(4)}$ Adding an attachment to the abutment teeth provides retention in addition to support, but this may be on the expense of the health of the Periodontium of the abutment teeth. ${ }^{(4)}$

Using attachments may lead to bone loss around the abutment teeth and increase their mobility due to the increased amount of unfavorable forces applied to them which may lead eventually to their loss. As a result, some prosthodontist tried to overcome this problem by providing the abutment teeth with copping without attachment mechanism to protect the abutment teeth from overloading and protect the Periodontium from any unfavorable forces. This treatment option showed long term survival periods but it doesn't provide retention to the prosthesis. ${ }^{(5)}$

\section{MATERIALS AND METHODS}

\section{Patient selection}

18 patient were selected from the outpatient clinic of the removable prosthodontics department, faculty of oral and dental medicine, Cairo University. Male patient with age range (45-70) and were medically free. The patient had few remaining teeth in the lower arch opposed by completely edentulous maxilla. All remaining teeth weren't mobile or had grade me mobility with sufficient zone of attached gingiva. Patient with a suitable inter-arch space to receive an overdenture with attachment. Angle's class I jaw relationship and No tempro-mandibular joint disorders.

\section{Patient examination}

In this stage the patients were checked if they matched the patient selection criteria of this research or not. Some diagnostic steps aided in the prosthetic stage as well.

Past and present medical and dental history were examined. Clinical examination

Was done intraoral and extra oral Extra-oral examination included assessing the patient's facial proportions and facial symmetry. Tempromandibular movements and function were examined. Thorough intraoral examinations were done for the remaining teeth, soft tissue and bone. Abutments mobility was checked by using the back side of two metal objects. The tooth mobility was given a grade from 0 till III according to Miller's classification. The residual ridge was examined for size, form and contour. The Amount of attached gingiva around abutment teeth and degree of gingival recession were measured. All the selected abutments teeth had a continuous zone of attached gingiva. Saliva was checked for consistency and amount. The frenal attachments and their relations to the sulcus and the ridge were examined. The size of the maxillary tuberosity and amount of undercut around them was considered during diagnosis. Also, the effect of the level of the floor of the mouth and movement on the denture prognosis was put in consideration. Panoramic radiographs were made for the patients to evaluate the bone and remaining tooth structure. Periapical radiographs using parallel technique for the proposed abutment teeth were taken to evaluate the supporting structures of the abutments. The proximal alveolar bone support should be more than half the length of the root of the abutment teeth on 
both sides (mesial or distal). The length of the root should allow for $9 \mathrm{~mm}$ for the post, $3 \mathrm{~mm}$ apical seal and $3 \mathrm{~mm}$ biological width and gingival crevice. Primary impressions were made for the upper and lower arches then Trial base and wax rim were constructed to be used for jaw relation records.

\section{Prosthetic phase}

The abutment teeth were shortened to a dome shape 2-3 $\mathrm{mm}$ above gum level and the root canal was prepared .Custom made trays were made on the primary cast and a Final impression was taken to the ridge and the abutments teeth with the prepared canals in one step. Border molding of the special tray was done using putty consistency addition silicon impression material for the lower arch. The plastic impression and burn out posts were inserted in the canals of the abutment teeth and their coronal parts were painted with adhesive to ensure they were picked up in the final impression. Single phase final impression was made using medium consistency addition silicon. The mandibular final impression was poured with type IV extra hard stone cast for post and core fabrication. Acrylic trial denture base and wax rim were constructed and used with Facebow to record the jaw relation.

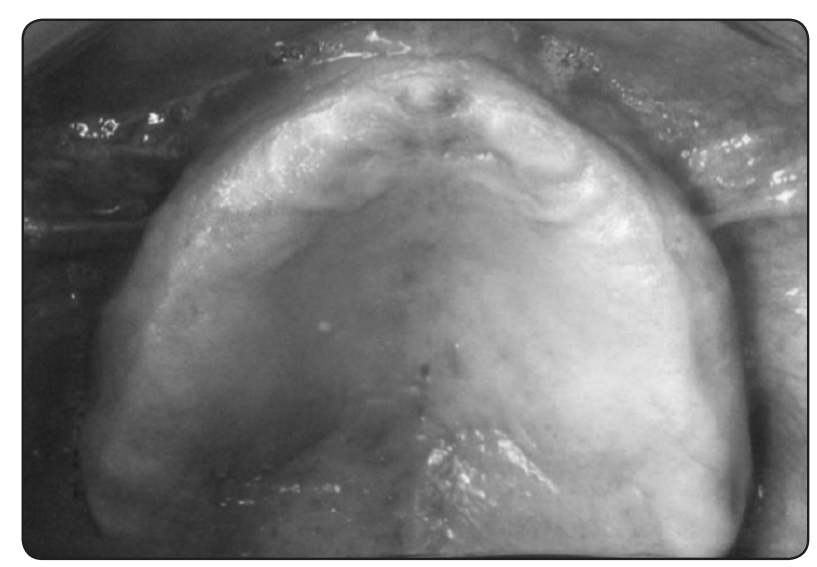

The patients were randomly divided into two equal groups, group A and group B

In group A the abutment teeth of the mandibular overdenture were covered by copping with a custom made designed low profile attachment. A special retention silicon was embedded in the lower acrylic denture base to act as housing for the attachment. In group B the abutment teeth of the mandibular overdenture received metal copings only without attachment. The attachments or the copings were seated in place and cemented with resin cement. The area around the attachment or the copping was relived to give enough space for the silicon material. The silicon primer was painted on the denture at the position of attachment to allow adhesion between the acrylic resin of the denture and the silicon pick-up material. The retention silicon material was mixed and intraoral relining was made with the denture base at the area of the attachment. After 20 min the denture base was removed and excess material was cut by a sharp lancet and the gingival margins of the abutment teeth were reliefed to prevent pressure on the gingival margins and mechanical irritation of the gingiva. Any further minor denture adjustments were made.

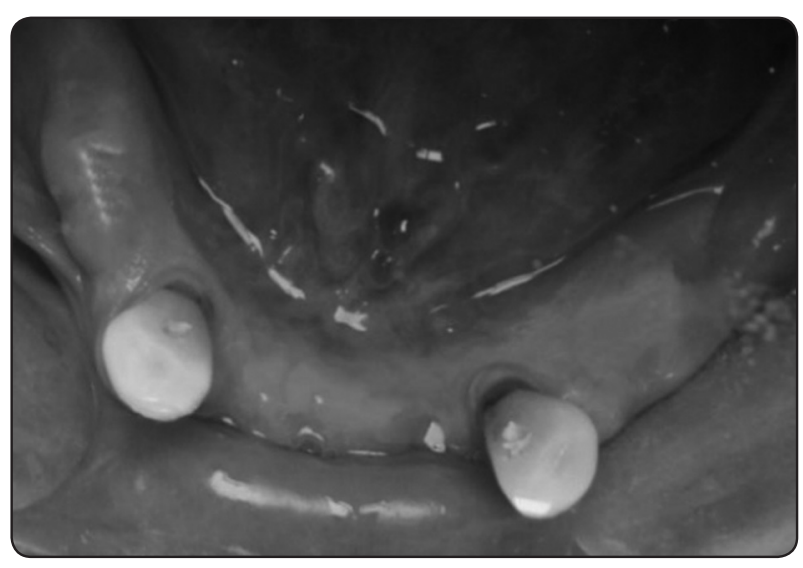

Fig. $(1,2)$ Upper and lower arch 
The upper and lower dentures and the area of biting of the film holder were painted with separating medium. Transparent Self-cure acrylic resin in the dough stage was added on the film holder and the patient was asked to bite on it with denture till complete setting of the acrylic resin. This step was made to put the film holder each time in the same place by the indentations of the denture teeth. Patients were evaluated radiographically to measure bone height end changes around the abutment teeth. The Digora computerized system, the Periapical parallel film holder and a specially constructed acrylic template were used for taking standardized and reproducible serial digital images using the long cone parallel technique. The film holder and the template ensure the position of the film to the abutment teeth as well as a fixed distance from the cone to the film in all the follow-up sessions. The distance were measured from the line perpendicular to the long axes of the tooth at the apex on both mesial and distal sides. This reading was used as the reference point for all the other follow up measurements after 3 and 6 months.
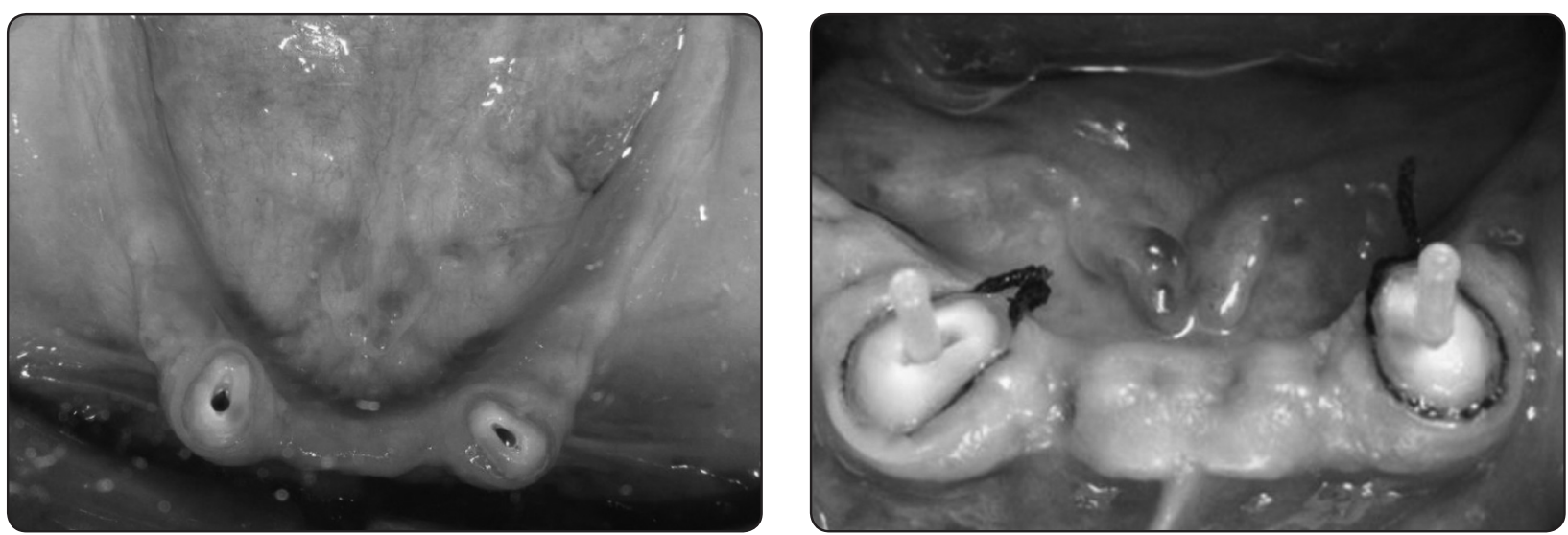

Fig. $(3,4)$ Lower arch preparation

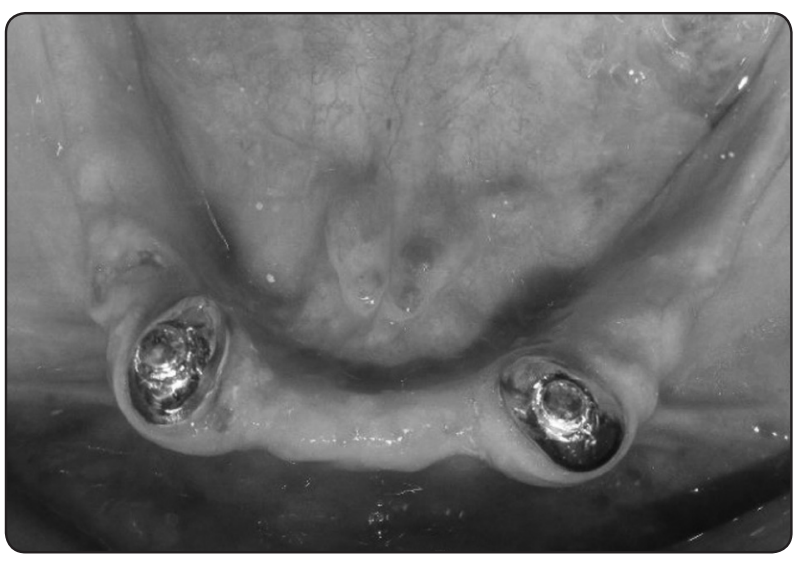

Fig. (5) Abutment with copping and attachment.

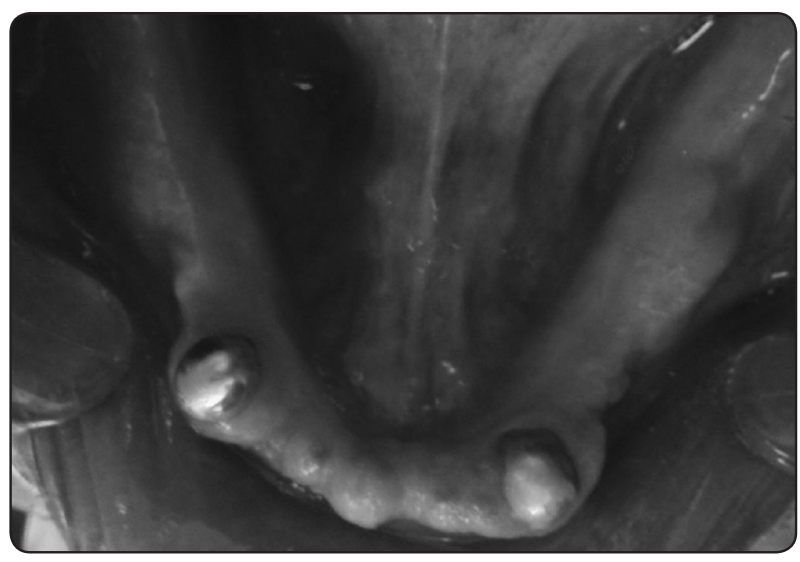

Fig. (6) Abutment with copping 


\section{RESULTS}

\section{A. Regarding group A (with attachment assembly):}

After calculation of mean difference of alveolar bone height for all surfaces of group A, it was revealed that there was decrease in alveolar bone height from time of insertion to three months by average $(0.46 \mathrm{~mm})$ as listed in table (1) and showed in figure (7). Also, there was decrease in alveolar bone height from three to six months by average $(0.46 \mathrm{~mm})$ as listed in table (1) and showed in figure (7). Finally, there was decrease in alveolar bone height from insertion to six months by average $(0.92 \mathrm{~mm})$ as listed in table (1) and showed in figure(7).

\section{B. Regarding group B (Coping only):}

After calculation of mean difference of alveolar bone height for all surfaces of group B, it was revealed that there was decrease in alveolar bone height from time of insertion to three months by average $(0.13 \mathrm{~mm})$ Also, there was decrease in alveolar bone height from three to six months by average $(0.17 \mathrm{~mm})$ Finally, there was decrease in alveolar bone height from insertion to six months by $(0.3 \mathrm{~mm})$.

\section{The comparison between the two groups}

The total amount of alveolar bone height was calculated for all surfaces for group A and group B which revealed respectively $(11.71,11.45)$ at time of insertion, $(11.25,11.32)$ at three months, $(10.79,11.15)$ at six months

TABLE (1) Means and standard deviations of alveolar bone height for all surfaces of group A and group B

\begin{tabular}{|c|c|c|c|c|}
\hline \multirow{2}{*}{ Follow up period } & \multicolumn{2}{|c|}{ GROUP A } & \multicolumn{2}{c|}{ GROUP B } \\
\cline { 2 - 5 } & Mean difference & Standard deviation & Mean difference & Standard deviation \\
\hline At time of insertion & 11.71 & 1.11 & 11.45 & 1.215 \\
\hline 3 months & 11.25 & 1.03 & 11.32 & 1.24 \\
\hline 6 months & 10.79 & 5.995 & 11.15 & 1.245 \\
\hline
\end{tabular}

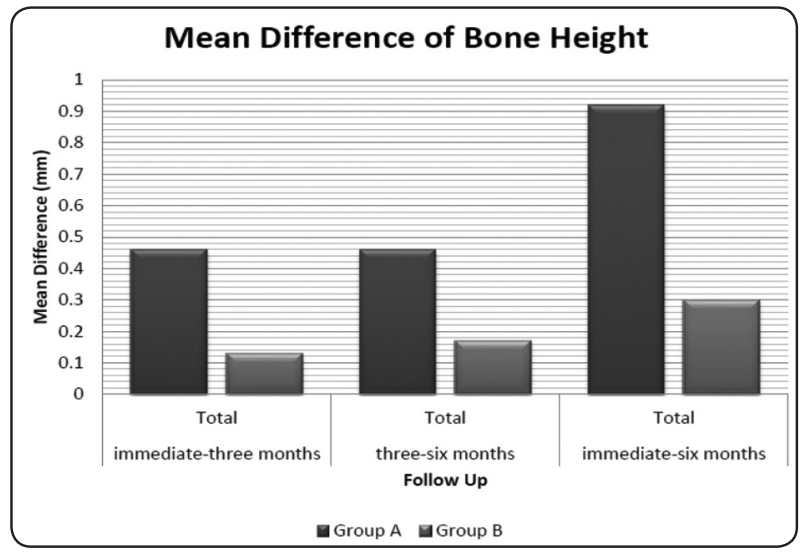

Fig. (7) Comparison of mean differences of alveolar bone height for all surfaces of group A and group B among follow up period

\section{DISCUSSION}

"It is more important to preserve what already exists than to replace what is missing" this statement represent one of the main concepts in modern dentistry. Today, with the more attention on preventive measures in prosthodontics the patients tend to choose overdenture instead of compete dentures .The patients have the opportunity to choose between adding an attachment or not over the abutment teeth in overdenture. In cases without attachment over the abutment teeth, the abutments provide mainly support and proprioception to the prosthesis. On the other hand, in case of adding an attachment to the abutment teeth extra retention 
is added to the overdenture. The extra retention obtained is accompanied with more stress on the abutment teeth which have an effect on the alveolar bone level of the abutments. In group A, patients received copping with attachment covering the abutment teeth of the mandibular overdenture and retention silicon material was used as housing. The results of this group showed statistical significant difference in alveolar bone loss around the abutment teeth throughout all the follow up periods. The amount of alveolar bone loss in the first follow up period from the beginning of the treatment to 3 months was relatively similar to the amount of alveolar bone loss in the follow up period from 3 months to 6 months. Radiographically, significant bone change was noted in the first 3 months after denture insertion of mean $0.46 \mathrm{~mm}$. Also, equal bone changes of $0.46 \mathrm{~mm}$ occurred from 3 to 6 months. The mean alveolar bone loss within the 6 months was $0.92 \mathrm{~mm}$. The study showed rapid bone changes around overdenture abutments in the first 6 months. ${ }^{(7)}$

The increase of bone resorption in the abutment teeth with the attachment assembly may be explained by the increased retention which is accompanied with more stresses on the abutment teeth. The alveolar bone respond to the increased stress by resorption.

In accordance with this, what was stated by A. B. Warren and Caputo 8 as they mentioned that there was a direct relationship between the stability and retention that each attachment design provided and the amount of stress and torque transferred to the supporting structures. Attachments that used parallelism or undercuts for retention tended to produce the most severe stress conditions on the supporting alveolus.

Also, the bone resorption may be related to the increased height of the abutment teeth due to presence of an attachment over the copping which increase the crown root ratio. With the increase in the crown root ratio there is an increase in the length of the lever arm which increase the amount of stress applied on the alveolar bone..$^{(9)}$

On the other hand, another reason may be related to the housing of the attachment itself. The silicon retention material was used as housing for the attachment by relining the denture base with it; that may result in a mechanism which depends only on the resiliency of the material for the stress breaking action. This may lead to unequal distribution of load between the abutment teeth and residual ridge. As the abutment teeth will carry more forces this will result in more stress transferred to the alveolar bone and consequently more bone resorption. ${ }^{(10)}$

In group B the patients received copping covering the abutment teeth of the mandibular overdenture. The results of this group showed less alveolar bone loss around the abutment teeth throughout all the follow up periods. Radiographically, little bone change was noted in the first 3 months after denture insertion of mean $0.13 \mathrm{~mm}$. An increase in bone loss of $0.17 \mathrm{~mm}$ occurred from 3 to 6 months. The mean alveolar bone loss within the 6 months was $0.3 \mathrm{~mm}$.

\section{CONCLUSION}

Within the limitation of this study, it can be concluded that using retention silicon material as housing with attachments doesn't reduce forces transmitted to the abutments to the level that eradicate alveolar bone resorption around the abutment teeth.

\section{REFERENCES}

1. The Glossary of Prosthodontic Terms. J. Prosthetic. Dent. 2005; 94(1):10-92.

2. Anselm E. Root-Retained Overdentures Part 1 Biomechanical and Clinical Aspects. J. Prosthetic. Dent. 1991.

3. Prince Ib. Conservation of the Supportive Mechanism. J. Prosthetic. Dent. 1965; 15:327-338.

4. Brkovic-Popovic S, Stanisic-Sinobad D, Djukanovic D, Postic S. Ten Years Longitudinal Study: Comparison of 
Abutment Alveolar Bone Levels of Overdenture Patients. Stomata. Glas. Srb. 2006; 53(1):17-26. Doi: 10.2298/ Sgs0601017b.

5. Pereira Jr. Overdentures and Masticatory Efficiency: Literature Review. J. Prosthetic. Dent. 7(4):34-38.

6. Sisodiya, Siddharth Drn. Tooth Supported Overdenture. Guide dent 2014; October.

7. Guglielmi F, Sônego F. User Satisfaction With Complete Dentures Made. Rgo, Rev Gaúch Odontol, Porto Alegre 2014; 62(2):123-128.
8. Muhammad A, Azad A, Azad Rest. Frequency of Complaints in Complete Denture Patients. Int.J.Dent. Clinics 2013; 12(5):1-4. Doi: 10.7326/M14-0180.

9. Rosslea Dm. Complete Denture Success for Patients and Dentists. Int. Dent. J. 2003; 53(5 Suppl):340-345

10. Gosavi Ss, Ghanchi M, Malik Sa, Sanyal P. A Survey of Complete Denture Patients Experiencing Difficulties with Their Prostheses. J. Contemp. Dent. Pact. 2013; 14(3):524527. Doi: 10.5005/Jp-Journals-10024-1355. 